\title{
Molecular Mechanism Underlying Pathogenesis of Lewisite-Induced Cutaneous Blistering and Inflammation
}

\section{Chemical Chaperones as Potential Novel Antidotes}

\author{
Changzhao Li, ${ }^{*}$ Ritesh K. Srivastava, ${ }^{*}$ Zhiping Weng, ${ }^{*}$ Claire R. Croutch, ${ }^{\dagger}$ Anupam Agarwal, ${ }^{\ddagger}$ Craig A. Elmets, ${ }^{*}$ Farrukh Afaq, ${ }^{*}$ \\ and Mohammad Athar*
}

From the Department of Dermatology* and the Division of Nephrology, ${ }^{\ddagger}$ Department of Medicine, University of Alabama at Birmingham, Birmingham, Alabama; and the Medical Countermeasures Division, ${ }^{\dagger}$ MRIGlobal Research Institute, Kansas City, Missouri

\author{
Accepted for publication \\ June 21, 2016. \\ Address correspondence to \\ Mohammad Athar, Ph.D., \\ Department of Dermatology, \\ University of Alabama at \\ Birmingham, 1530 3rd Ave. \\ S., VH 509, Birmingham, AL \\ 35294-0019. E-mail: mathar@ \\ uab.edu.
}

\begin{abstract}
Lewisite is a potent arsenic-based chemical warfare agent known to induce painful cutaneous inflammation and blistering. Only a few modestly effective antidotes have so far been described in the literature. However, the discovery of effective antidotes for lewisite was hampered by the paucity of the exact molecular mechanism underlying its cutaneous pathogenesis. We investigated the molecular mechanism underlying lewisite-induced cutaneous blistering and inflammation and describe its novel antidotes. On the basis of our initial screening, we used a highly sensitive murine model that recapitulates the known human pathogenesis of arsenicals-induced cutaneous inflammation and blistering. Topically administered lewisite induced potent acute inflammation and microvesication in the skin of Ptch $1^{+/-} /$SKH-1 mice. Even at a very low dose, lewisite up-regulates unfolded protein response signaling, inflammatory response, and apoptosis. These cutaneous lesions were associated with production of reactive oxygen species and extensive apoptosis of the epidermal keratinocytes. We confirmed that activation of reactive oxygen species-dependent unfolded protein response signaling is the underlying molecular mechanism of skin damage. Similar alterations were noticed in lewisitetreated cultured human skin keratinocytes. We discovered that chemical chaperone 4-phenyl butyric acid and antioxidant $\mathrm{N}$-acetylcysteine, which significantly attenuate lewisite-mediated skin injury, can serve as potent antidotes. These data reveal a novel molecular mechanism underlying the cutaneous pathogenesis of lewisite-induced lesions. We also identified novel potential therapeutic targets for lewisite-mediated cutaneous injury. (Am J Pathol 2016, 186: 2637-2649; http://dx.doi.org/10.1016/ j.ajpath.2016.06.012)
\end{abstract}

Lewisite [dichloro (2-chlorovinyl) arsine] is a potent arsenical vesicant chemical warfare agent with significant systemic toxicity. ${ }^{1}$ It was first synthesized in 1904 and later rediscovered by Captain W. Lee-Lewis in 1918 in the United States. Although it was proposed for use as a chemical weapon, fortunately it was never applied to the battlefield. ${ }^{2}$ Nonetheless, it is known that several countries, including Germany, Italy, the United States, Russia, and Japan, have stockpiled significant amounts of lewisite, causing a significant concern for public health. Being
Supported by CounterACT Program, NIH, Office of the Director, and National Institue of Arthritis and Musculoskeletal and Skin Diseases grants R21 AR064595 and UO1 NS095678 (M.A.).

C.L. and R.K.S. contributed equally to this work.

NIH had no involvement in the study design; collection, analysis, and interpretation of data; the writing of the manuscript; or the decision to publish the manuscript.

Disclosures: None declared.

A guest editor acted as the editor-in-chief for the manuscript. No person at the University of Alabama at Birmingham was involved in the peer review or final disposition of this article. 
highly toxic and quick-acting vesicant chemicals, lewisite and other structurally related arsenicals, such as methyldichloroarsine, phenyldichloroarsine, and ethyldichloroarsine, have always been considered to be potential candidates for chemical weapons. ${ }^{1}$ Unintentional exposure or intentional use by terrorists could be another significant threat. Other analogs of lewisite, such as phenylarsine oxide (PAO), phenydiidoarsine, trans-chlorovinylarsine oxide, and transchlorovinyldiiodide, manifest similar but less severe effects in murine skin and human skin xenograft. ${ }^{4}$

Lewisite can burn and blister any part of the body it comes in contact with. It is considered much more reactive than mustard gas. ${ }^{5}$ If not decontaminated effectively and immediately, 30 drops $(2.6 \mathrm{mg})$ of lewisite exposed to the skin can be lethal. ${ }^{2}$ The death may be caused by its systemic toxicity from "lewisite shock," which is severe fluid loss and hypovolemia secondary to capillary leakage. ${ }^{6}$ Besides skin, eyes and respiratory tract are the most likely targets of lewisite. $^{2}$ The physicochemical characteristics of lewisite that include its lipophilic nature make it penetrate the skin rapidly. Topically exposed lewisite induces acute inflammation associated with severe pain, which develops within 10 to 12 seconds, followed by erythema, edema, and blistering, which appear later. ${ }^{7}$ Its reactivity with glutathione, leading to its loss followed by a decrease in overall protein thiols, was considered the major mechanism for these manifestations. Dysregulation of calcium homeostasis due to oxidative stress, lipid peroxidation, and membrane damage, leading to cell death, is also described. ${ }^{1}$

Early strategies that led to the development of British anti-Lewisite (BAL) as its antidote are based on the arsenic chelating properties of BAL. Indeed, BAL treatment had some efficacy in reducing lewisite-induced tissue damage. ${ }^{8}$ However, BAL is a toxic compound that has very low solubility in water. Its treatment requires painful intramuscular injections. ${ }^{9}$ Water soluble analogs of BAL have less toxicity compared with BAL but reduced efficacy against lewisite-induced skin lesions in humans. ${ }^{9}$ Thus, other than BAL, largely there is no effective US Food and Drug Administration-approved therapeutic approach to reduce lewisite toxicity. Therefore, developing novel, more efficacious therapeutic drugs for counteracting lewisite-induced toxicity will largely depend on defining its molecular pathogenesis.

Endoplasmic reticulum (ER) is the site of biosynthesis, assembly, folding, and maturation of many secretory and membrane-bound proteins. Disruption of ER homeostasis may result in the accumulation of unfolded and/or misfolded proteins, leading to the condition known as ER stress. On ER stress, unfolded protein response (UPR) signaling is activated. The UPR pathway regulates the biosynthesis of chaperone proteins. These chaperone proteins bind with partially folded or unfolded proteins to restore the proteinfolding capacity of ER and provide a balance between protein-folding overload and impaired ER capacity. This is achieved by engaging three ER membrane resident proteins:
PERK, IRE1, and ATF6. However, prolonged activation of UPR signaling may lead to the pathogenesis of inflammation and tissue damage by inducing cell death. UPR-regulating proteins have also been associated with multiple other conditions, such as neurodegenerative and metabolic disorders and tumorigenesis. ${ }^{10}$

The interplay of reactive oxygen species (ROS) and ER stress is also found under certain experimental conditions. ${ }^{11}$ Because at least some of the systemic effects of lewisite are thought to be related to arsenic toxicity, in this study we explored the mechanism by which lewisite induces acute cutaneous inflammation and tissue damage. We believe that arsenic could play an important role in the manifestation of lewisite toxicity. Our data indeed indicate that topical challenge of lewisite onto the skin of $P t c h 1^{+/-} / S K H-1$ mice and treatment of human keratinocytes with lewisite activate the UPR-signaling pathway, inflammatory responses, and cell death, suggesting a role of UPR signaling in lewisitemediated tissue injury. Interestingly, treatment with the chemical chaperone 4-phenylbutyric acid (4-PBA) afforded protection against lewisite-induced inflammation and tissue injury by blocking the UPR-signaling pathway. These data strengthen the role of this pathway in lewisite toxicity. We also found upstream involvement of ROS in triggering lewisite-mediated skin damage. Consistently, the observation that the antioxidant $\mathrm{N}$-acetylcysteine (NAC) affords significant protection against lewisite toxicity suggests that ROS-regulated UPR signaling is one of the underlying key molecular pathways of lewisite cutaneous toxicity. Therapeutic approaches targeting UPR signaling and ROS may lead to the development of novel and highly effective antidotes against lewisite-induced tissue damage.

\section{Materials and Methods}

\section{Materials}

Lewisite was synthesized by MRIGlobal Research Institute (Kansas City, MO). PAO (P3057), NAC (A7250), and 4-PBA (P21005) were from Sigma-Aldrich (St. Louis, MO). CMH2DCFDA (C6827) was from Life Technology (Carlsbad, CA). Immortalized human keratinocytes, HaCaT cells (T0020001), was from AddexBio Technologies (San Diego, CA). SSoFast Eva Green Supermix (172-5202) was from BioRad (Hercules, CA). Antibodies cleaved caspase-3 (9664), phospho-eIF2 $\alpha$ (3398s), eIF2 $\alpha$ (9722), CHOP (2895), ATF4 (11815s), phopho-JNK1/2 (9251), and phospho-c-Jun (9261) were from Cell Signaling (Danvers, MA); GRP78 (sc-1050) and phospho-I $\kappa \mathrm{B} \alpha$ (sc-101713) were from Santa Cruz (Dallas, TX); phospho-NF-кB p65 (ab30623) and IL-1 $\beta$ (ab9722) were from Abcam (Cambridge, UK); cyclooxygenase 2 (COX2) (160126) was from Cayman Chemical (Ann Arbor, MI); F4/80 (14-4801-82) was from eBioscience (San Diego, CA); and CD11b (BD557395) and Gr1 (BD553126) were from BD (San Jose, CA). Cignal Finder 45-Pathway Reporter Array plate (CCA-901L), RT ${ }^{2}$ First Strand kit (330401), RT ${ }^{2}$ qPCR Master 
Mix (330523), and mouse inflammatory cytokines and receptor PCR arrays (PA-011-12) were from Qiagen (Hilden, Germany). Dual-Glo Luciferase Assay System (E2940) and CellTiter 96 AQueous One Solution Cell Proliferation Assay (G3582) were from Promega (Madison, WI). Prostaglandin $\mathrm{E}_{2}\left(\mathrm{PGE}_{2}\right) \mathrm{Ex}-$ press Enzyme Immunoassay Kit (500141) was from Cayman Chemical. In situ Cell Death Detection Kit (1684795) was from Roche (Indianapolis, IN). Real-time quantitative PCR (qPCR) primers mIllb forward 5'-AAAGCCTCGTGCTGTCGGACC-3' ${ }^{\prime}$, reverse 5'-CAGGGTGGGTGTGCCGTCTT-3'; mIl6 forward 5'-GGTGACAACCACGGCCTTCCC-3', reverse 5'-AAGCCTCCGACTTGTGAAGTGGT-3'; mTnf forward 5'-AGCCCACGTCGTAGCAAACCAC- $3^{\prime}$, reverse $5^{\prime}$-TCGGGGCAGCCTTGTCCCTT-3'; mTgfb1 forward 5'-CGGCTGCTGACCCCCACTGA-3', reverse 5'-ACGTTTGGGGCTGATCCCGTT- $3^{\prime}$ and mGapdh forward $5^{\prime}$-CAATGTGTCCGTCGTGGATCT- ${ }^{\prime}$, reverse 5'-GTCCTCAGTGTAGCCCAAGATG- ${ }^{\prime}{ }^{\prime}$ were obtained from Invitrogen (Carlsbad, CA).

\section{Animals}

$C 57 B L / 6$ and $F V B$ mice were purchased from the Jackson Laboratory (Bar Harbor, ME). Ptchl ${ }^{+/} / S K H-1$ hairless mice were generated by crossing Ptch $1^{+/} / C 57 B L / 6 J$ into SKH-1 background. ${ }^{12}$ Mice recruited in the current study represented the 35th generation, which has almost pure $S K H-1$ background.

\section{Topical Treatment of Lewisite to Animals}

Topical application of lewisite to $P t c h 1^{+/-} / S K H-1$ hairless mice (aged 5 to 6 week) were performed by MRIGlobal. All protocols were approved by Institutional Animal Care and Use Committee of University of Alabama at Birmingham and MRIGlobal. Before lewisite administration, mice were anesthetized with $100 \mathrm{mg} / \mathrm{kg}$ of ketamine and 5 to $7 \mathrm{mg} / \mathrm{kg}$ of xylazine by i.p. injection. To manage pain, 0.05 to $0.1 \mathrm{mg} / \mathrm{kg}$ of buprenorphine was administered 30 minutes before anesthesia and again every 8 to 12 hours until termination of the experiment. An indelible marker was used to mark the dosing site area. Lewisite diluted in $30 \mu \mathrm{L}$ of ethanol was administered topically on the dorsal skin of $P t c h 1^{+/} / S K H-1$ hairless mice (6 mice per group) at different doses (vehicle only, $0.75 \mathrm{mg} / \mathrm{kg}, 1.5 \mathrm{mg} / \mathrm{kg}$, and $2.25 \mathrm{mg} / \mathrm{kg}$ in an area of $2 \mathrm{~cm}^{2}$ ). For therapeutic study, lewisite was applied in a square of $1.6 \times 1.6 \mathrm{~cm}^{2}$ with a dose of $2.0 \mathrm{mg} / \mathrm{kg}$ as determined by the dose dependent study previously described. Thereafter, $5 \mathrm{mg}$ of NAC per mouse or $4 \mathrm{mg}$ of 4-PBA per mouse was applied topically to the dose-site skin within 5 to 10 minutes after lewisite or vehicle exposure. A universal vehicle (mixture of one-third $\mathrm{H}_{2} \mathrm{O}$, one-third ethanol, and one-third glycerol) was formulated to easily dissolve both 4-PBA and NAC. In all these experiments, clinical observations, skin bifold thickness, and images of dose-site skin were recorded at different intervals after exposure. At the termination of the experiment, all mice were euthanized, and skin tissue samples were collected for further analysis. Decontaminated skin samples were shipped to Dr. Athar's laboratory at University of Alabama at Birmingham.

\section{Treatment of Cells with Lewisite}

Lewisite in vitro studies were performed by MRIGlobal. HaCaT cells were maintained in Dulbecco's modified Eagle's medium (10\% fetal bovine serum, $1 \%$ antibiotics) at $37^{\circ} \mathrm{C}$ in $5 \% \mathrm{CO}_{2}$ incubator. Cells were plated in 6-well plates and were allowed to reach $80 \%$ confluency before treatment. Lewisite stock was diluted in Dulbecco's modified Eagle's medium to final concentrations before the exposure. Then growth medium was replaced with $2 \mathrm{~mL}$ of medium containing various concentrations of lewisite for 6 hours. Protein lysates were collected for Western blot analysis. For cell viability assay, the CellTiter 96 AQueous One Solution Reagent containing a compound 3-(4, 5-dimethylthiazol-2-yl)-5-(3-carboxymethoxyphenyl)-2-(4sulfophenyl)-2H-tetrazolium (MTS) was used. The amount of soluble MTS formazan produced by cellular reduction of MTS is measured by absorbance at $490 \mathrm{~nm}$ which represents the number of living cells in culture. Cells treated with 0.5 , 1 , and $2 \mu \mathrm{mol} / \mathrm{L}$ lewisite or in the presence of $10 \mathrm{mmol} / \mathrm{L}$ $\mathrm{NAC}$ or $1 \mathrm{mmol} / \mathrm{L}$ 4-PBA were incubated with $300 \mu \mathrm{L}$ of MTS for 3 hours after which reading of absorbance at 490 nm was performed.

\section{Cell-Based Multiple-Pathway Activity Assay}

Multiple-pathway activity assay was performed in $\mathrm{HaCaT}$ cells using Cignal Finder 45-Pathway Reporter Array plate. A reverse transfection protocol was used according to the manufacturer's instructions. After 24 hours, cells were treated with $200 \mathrm{nmol} / \mathrm{L}$ PAO for 6 hours. The luciferase assay was performed using the Dual-Glo Luciferase Assay System following the manufacturer's protocol. The results were analyzed by online data analysis software from Qiagen (Data Analysis 45 Report Array software, http://www.sabiosciences.com/reporter_assay_ product/HTML/CCA-901L.html\#resource, last accessed August 24, 2013).

\section{PCR Array}

PCR array was performed using the PCR Array system (SABiosciences, Frederick, MD) as described earlier. ${ }^{13}$ Briefly, first-strand cDNA synthesis was performed using $\mathrm{RT}^{2}$ First Strand kit (SABiosciences). PCR was performed with mouse inflammatory cytokines and receptor PCR Arrays on a 7500 fast real-time PCR system (Applied Biosystems, Foster City, CA) using $\mathrm{RT}^{2}$ qPCR Master Mix. The program was $95^{\circ} \mathrm{C}$ for 10 minutes, followed by 40 cycles of $95^{\circ} \mathrm{C}$ for 15 seconds, and $60^{\circ} \mathrm{C}$ for 1 minute. For 

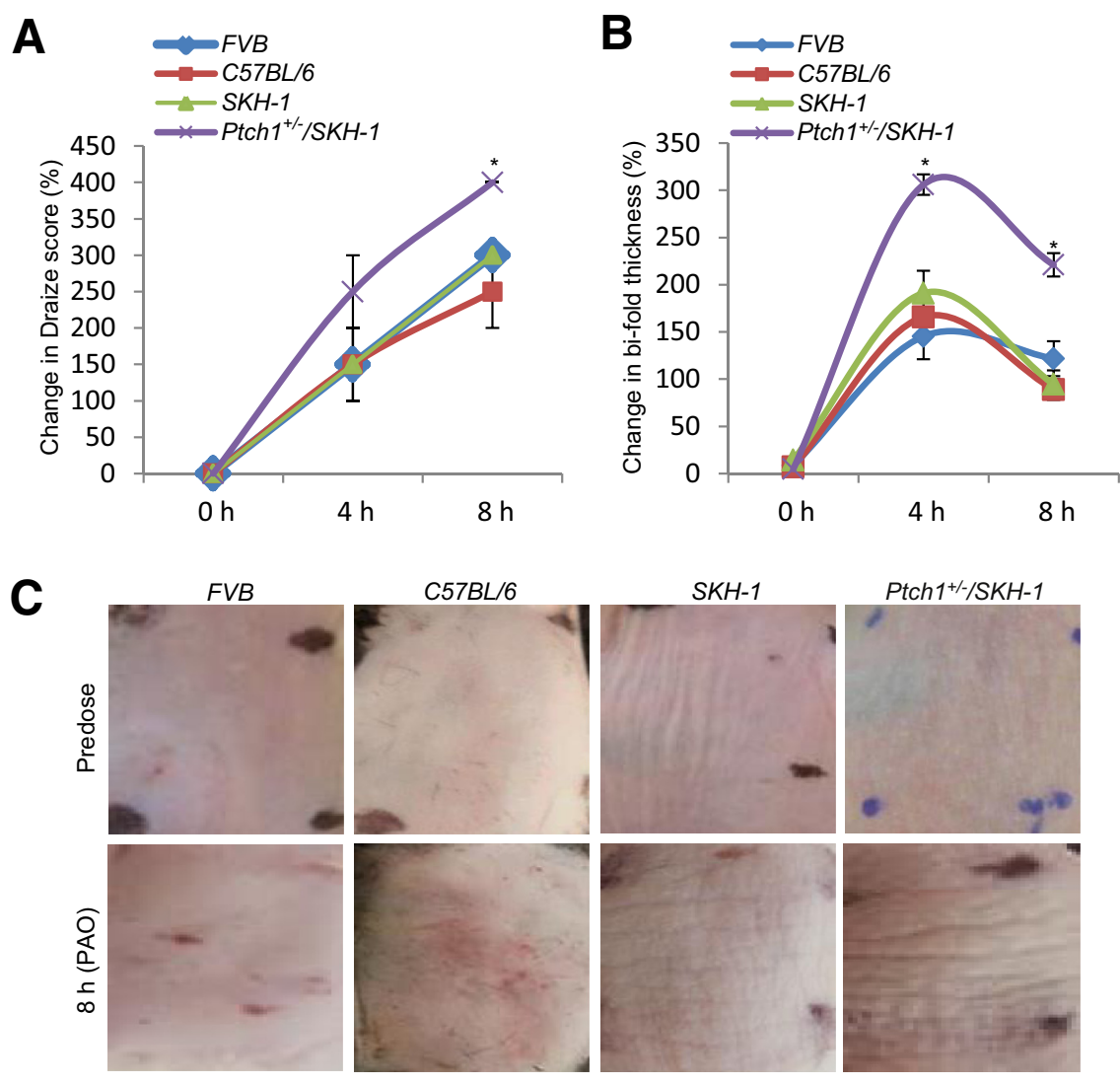

Figure 1 Selection of a murine model showing high sensitivity to phenylarsine oxide (PAO)induced cutaneous inflammation. FVB, C57BL/6, SKH-1, and $P$ tch $1^{+/-} /$SKH-1 mice were selected for assessing their sensitivity to PAO topical treatment. PA0 preparations and treatment $(100 \mu \mathrm{g}$ per mouse) were performed in continuously operated chemical and biological hood according to standard operation procedure approved by the Institutional Animal Care and Use Committee of the University of Alabama at Birmingham. A and B: Graph showing the percentage of changes in Draize score and skin bifold thickness. C: Comparison of the gross changes of PA0-exposed skin (within the marked area) at 0 and 8 hours after treatment. Data are expressed as means \pm SEM of three animals. ${ }^{*} P<0.05$ versus $F V B, C 57 B L / 6$, or SKH-1 mice. each group, four skin samples were pooled and subjected to PCR Array analysis. Relative fold-changes of gene expression were calculated and heat map was generated by web-based software provided by the manufacturer $\left(\mathrm{RT}^{2}\right.$ Profiler PCR Array Data Analysis version 3.5; SABiosciences, http://pcrdataanalysis.sabiosciences.com/pcr/ arrayanalysis.php, last accessed June 13, 2016).

\section{qPCR Analysis}

qPCR analysis was performed in SSoFast SYBR Green PCR master mix. Cycling conditions were 20 seconds at $95^{\circ} \mathrm{C}$ followed by 40 cycles at $95^{\circ} \mathrm{C}$ for 3 seconds and $60^{\circ} \mathrm{C}$ for 30 seconds. Relative quantification of target mRNA levels was calculated using $2\left(-\Delta \Delta \mathrm{C}_{\mathrm{T}}\right)$ method. Gapdh was used as an endogenous reference gene.

\section{Immunohistochemistry, Immunofluorescence, and Western Blot}

These assays were performed as described previously. ${ }^{12,13}$

\section{Determination of ROS in the Skin}

Detection of ROS in the skin of $P t c h 1^{+/-} / S K H-1$ mice was performed using CM-H2DCFDA as described previously. ${ }^{13}$

\section{TUNEL Assay}

Terminal deoxynucleotidyl transferase-mediated dUTP nick-end labeling (TUNEL) staining was performed using the in situ Cell Death Detection kit from Roche according to the manufacturer's standard protocol.

\section{Enzyme-Linked Immunosorbent Assay}

Enzyme-linked immunosorbent assay was performed using the $\mathrm{PGE}_{2}$ Express Enzyme Immunoassay Kit according to the manufacturer's instructions (Cayman Chemical). Data were analyzed by the spreadsheet from Cayman Chemical.

\section{Statistical Analysis}

Statistical analysis between the two groups was performed using the $t$-test. $P<0.05$ was considered statistically significant.

\section{Results}

Screening for Murine Model of Arsenicals-Induced Cutaneous Inflammation and Blistering

Because limited studies have been conducted in animal models to investigate the pathogenesis of lewisite-induced skin injury, ${ }^{9,14}$ we developed a suitable murine model that 


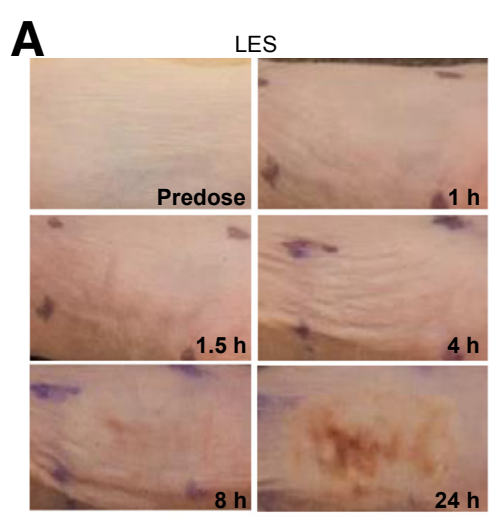

B

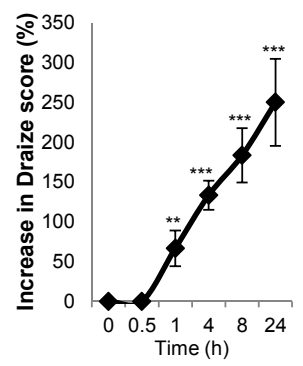

E

$\mathbf{F}$
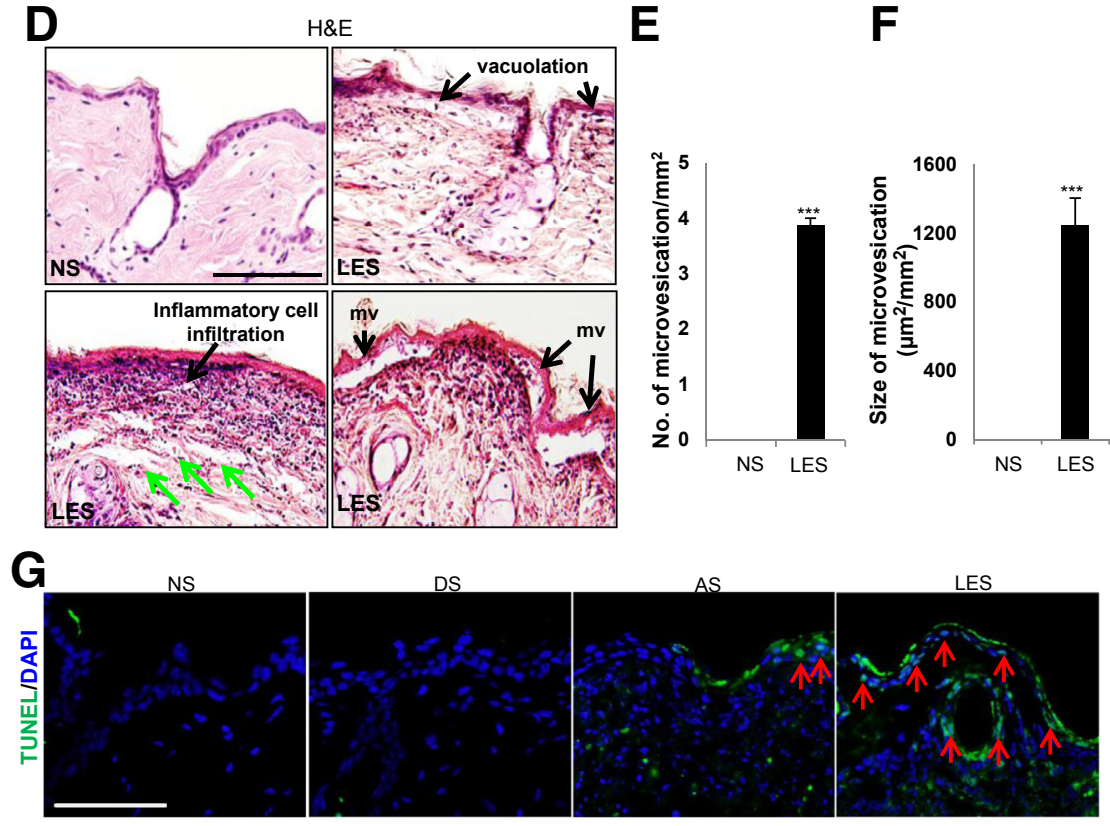

H

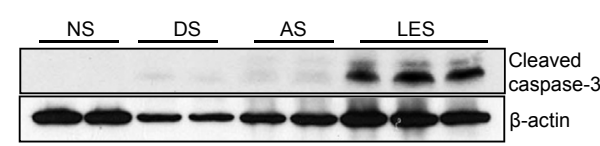

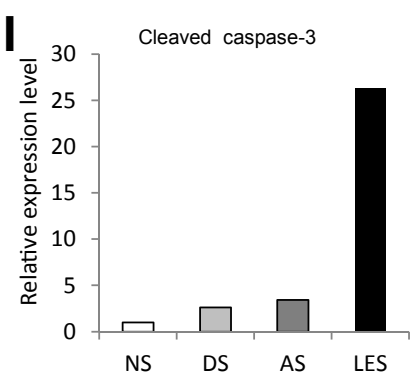

Figure 2 Topical administration of lewisite induces acute inflammation and skin damage in Ptch $1^{+/-} /$SKH-1 mice. The skin samples analyzed here were from the experiment in which $1.5 \mathrm{mg} / \mathrm{kg}$ of lewisite was applied on the skin of a $2-\mathrm{cm}^{2}$ area. A: Images showing the time-dependent effect of lewisite exposure on the induction of cutaneous lesions. Representative images of male mice are shown. B and C: Clinical observations of lewisiteexposed skin (LES) at different time points were recorded using a Draize scoring system. Graphs showing percentage of change in Draize score and skin bifold thickness at different intervals. D: Histologic examination of skin sections showing lewisite-induced skin damages and inflammation. Green arrows indicate the separation of collagen bundles by edema fluid in the dermis of LES. $E$ and F: Graphs showing the number and size of microvesication per unit area. G: Representative images of terminal deoxynucleotidyl transferase-mediated dUTP nick-end labeling (TUNEL) staining in the skin. Red arrows indicate TUNEL-positive keratinocytes in the skin. $\mathbf{H}$ and $\mathbf{I}$ : Western blot and densitometry analysis of the expression of cleaved caspase-3. Data are expressed as means \pm SEM of six animals (B, C, E, F). Data are presented as mean only (I). ${ }^{* *} P<0.01,{ }^{* *} P<0.001$ versus 0 hour. Scale bars $=50 \mu \mathrm{m}$ (D and $\mathbf{G})$. AS, adjacent skin; DS, distal skin; H\&E, hematoxylin and eosin; mv, microvesication; NS, nonexposed skin. can recapitulate the effects of lewisite in human skin. It is known that $C 57 B L / 6$ (haired) is resistant, whereas $F V B$ (haired) and $S K H-1$ (hairless) are sensitive mouse strains to cutaneous chemical injury. ${ }^{15}$ In addition, we developed a mouse model $P t c h 1^{+/-} / S K H-1$, which is highly sensitive to cutaneous inflammation, particularly induced by UVB. ${ }^{12}$ Therefore, we used these mouse strains to evaluate their relative sensitivity to arsenicals. A lewisite analog, PAO, was used for these studies ${ }^{4}$ because we consider it a surrogate chemical for other highly toxic arsenicals based on our in vitro and in vivo investigations (R.K. Srivastava, C. Li, Z. Weng, A. Agarwal, C.A. Elmets, F. Afaq, and M.
Athar, unpublished data). For initial assessment, we conducted a number of investigations using this agent. Topical treatment of skin of various mouse strains with PAO was performed in an identical fashion. The cutaneous injury and inflammation as assessed by evaluating overall skin injury (Draize scoring) (Supplemental Table S1) and measurement of skin bifold thickness (edema) at 4 and 8 hours after treatment. We found that $P t c h 1^{+/-} / S K H-1$ was more sensitive or at least comparable to other mouse strains for PAOinduced skin injury (Figure 1). Thus, $\mathrm{Ptchl}^{+/-} / \mathrm{SKH}-1$ mice have significantly higher Draize score at both 4 and 8 hours after PAO treatment compared with $C 57 B L / 6, F V B$, and 
A

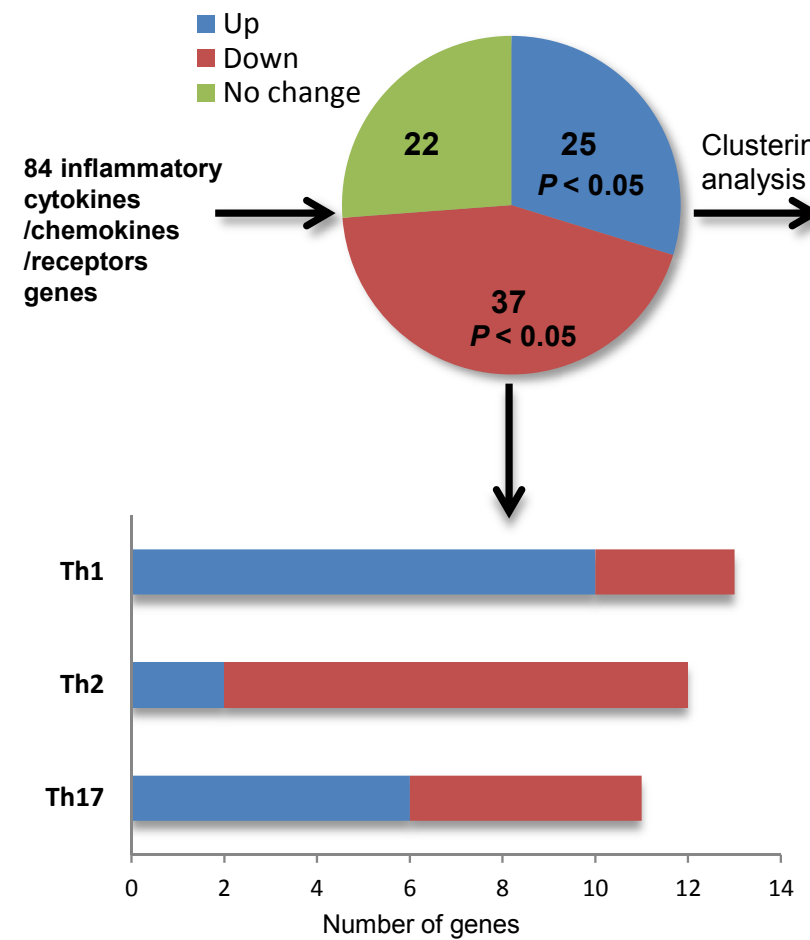

B
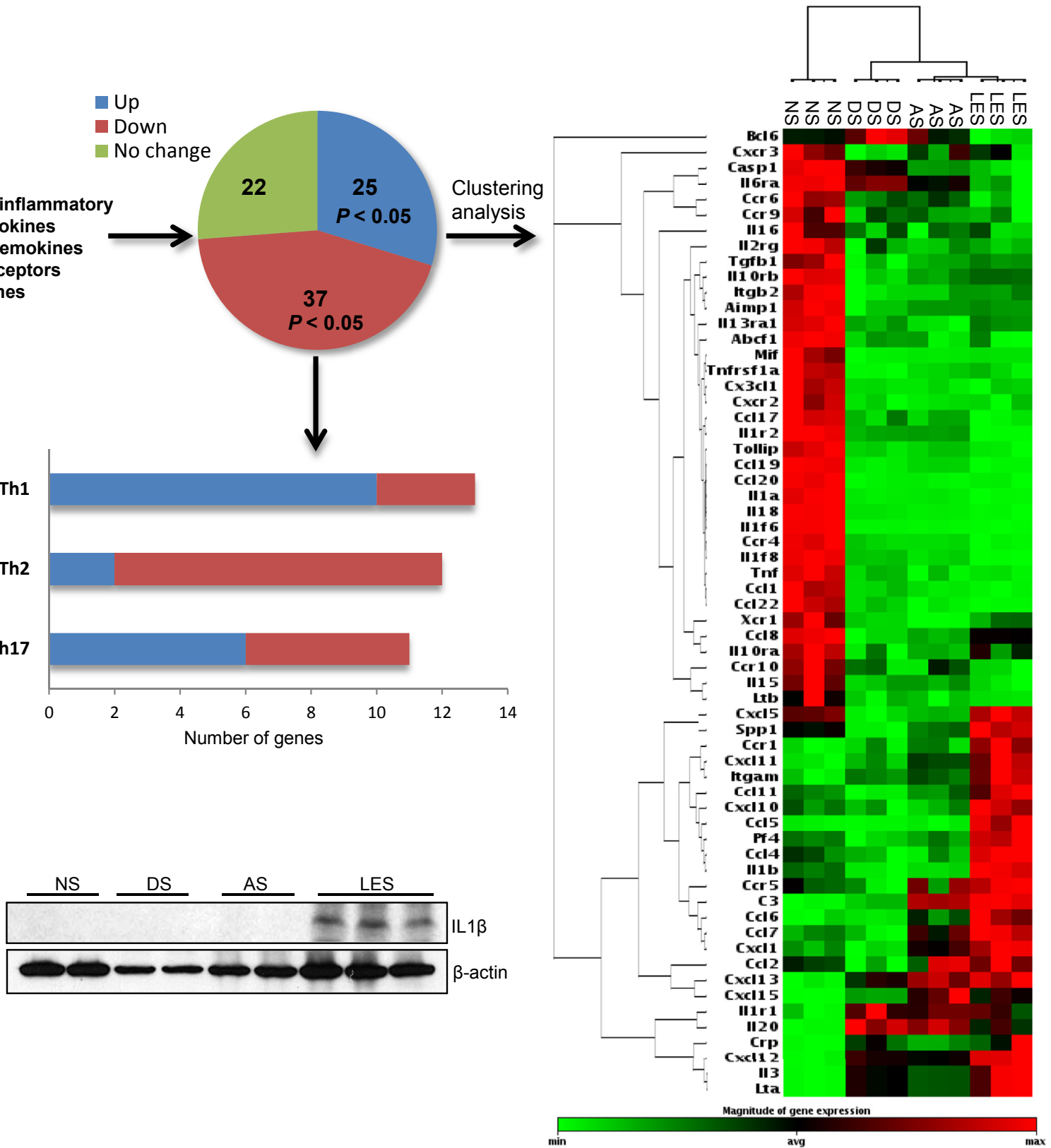

C
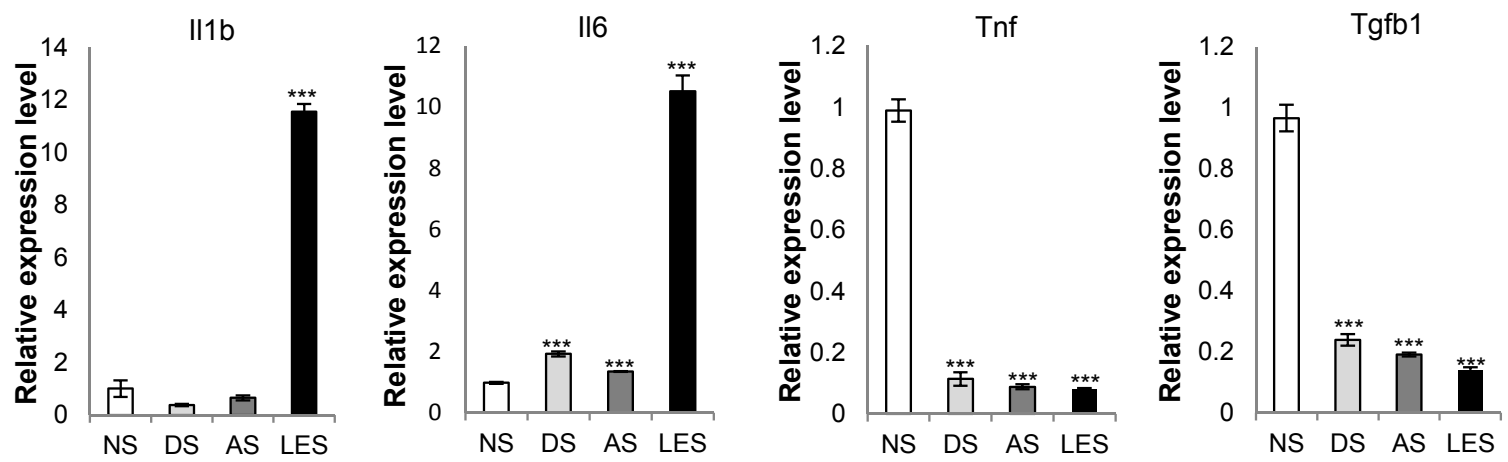
SKH-1 mice (Figure 1A). Similarly, PAO caused more robust increase in skin bifold thickness in $P t c h 1^{+/-} / S K H-1$ mice than in other mouse strain tested (Figure 1B). In Ptchl ${ }^{+/} / S K H-1$ mice, edema and erythema could be observed 1 hour after exposure, and their responses became severe at 4 hours. After 8 hours, the edematous area became gray-white, but skin bifold thickness remained significantly increased (Figure 1C). These gross skin alterations in response to PAO challenge were comparable to those reported in human skin exposed to lewisite and another lewisite analog, phenyldichloroarsine, which had similar vesicant potency to that of lewisite. ${ }^{4}$ On the basis of these observations, we selected $P t c h 1^{+/} / S K H-1$ as our murine model to define the pathogenesis of lewisite-induced skin lesions.

\section{Lewisite Causes Intensive Cutaneous Damage and Acute Inflammatory Responses in the Skin of Ptch $^{+/-} /$SKH-1 Mice}

After treatment with lewisite, mild erythema and edema were developed at the dosing site as early as 1 hour after exposure. By 4 hours to 8 hours after exposure, edema was severe and more clearly visible, whereas erythema disappeared and the skin lesions acquired grayish color (Figure 2A). Qualitatively, early macroscopic skin manifestations were consistent with those observed in lewisite-exposed $S K H-1$ mice. ${ }^{14}$ Measurement of skin bifold thickness revealed a persistent increase in edema from 8 hours to 24 hours, which was accompanied by the eventual development of dark brown skin color of lesions at least in some focal areas. Consistently, a time-dependent increase in Draize score was highly significant from 1 hour to 24 hours after exposure (Figure 2, A-C).

Hematoxylin and eosin staining of the lesional skin sections revealed advanced epidermal degeneration and infiltration of inflammatory cells in the underlying dermis (Figure 2D). In the epidermis, morphologic changes in nuclei of keratinocytes were noticed, which were characterized by pyknosis, karyorrhexis, and karyolysis in necrotic area. Cells residing in hair follicles and sebaceous glands had similar alterations (Figure 2D). Increased cytoplasmic eosinophilia and occasional vacuoles formation in the epidermis were also observed (Figure 2D). A larger number of subepidermal microvesications were noticed in lewisitetreated skin. These subepidermal microvesications were closely associated with the large number of inflammatory cells infiltration (Figure 2, D-F). Accumulation of interstitial edema fluid was indicated by separation of dermal collagen bundles (Figure 2D). TUNEL staining revealed that not only the epidermal skin keratinocytes but also the hair follicular and dermal cells were apoptotic or necrotic, particularly around dosing site (Figure 2G). Although cell death could also be found in lesion-adjacent skin, these changes were uncommon in the distal skin areas as also confirmed by Western blot analysis of cleaved caspase- 3 (Figure 2, H and I). Importantly, these macroscopic and microscopic skin alterations were identical to those previously described in human skin and in other animal model (white pig) as well. ${ }^{4,16}$ Notably, the same dose of lewisite applied to different areas of the skin causes variations in the damage quantitatively and qualitatively. However, detailed assessment of these changes is beyond the scope of this article.

\section{Characterization of Lewisite-Induced Cutaneous Inflammatory Responses in Ptch $1^{+/-} / \mathrm{SKH}-1$ Mice}

We characterized the inflammatory milieu by immunofluorescence staining of various surface markers on inflammatory cells. Lewisite treatment induces infiltration of neutrophils $\left(\mathrm{CD} 11 \mathrm{~b}^{+} / \mathrm{Gr}^{+}\right)$and macrophages (F4/ $80^{+}$) 24 hours after exposure (Supplemental Figure S1). To better characterize the lewisite-induced cutaneous inflammation, we used PCR Array that contained 84 inflammation-associated genes and assessed the gene expression profile for nonexposed skin, distal skin, adjacent skin, and lewisite-exposed skin. Lewisite significantly up-regulated the expression of 25 genes but down-regulated the expression of 37 genes in lewisiteexposed skin when compared with nonexposed skin (Figure 3A). These genes were further subjected to clustering analysis using online RT Profiler PCR Array data analysis software version 3.5 (SABiosciences). The clustering patterns of these samples fell into two major groups. Nonexposed skin samples were clustered in one group, whereas lewisite-exposed skin, adjacent skin, and distal skin were clustered in the other group in which

\footnotetext{
Figure 3 Characterization of lewisite-induced inflammation in the skin of $P t c h 1^{+/-} /$SKH-1 mice. A: PCR Array data showing the gene expression profile of 84 inflammatory cytokines, chemokines, or receptors. Of these 84 genes, 25 genes were found to be up-regulated $(P<0.05)$, whereas 37 genes were downregulated $(P<0.05)$ after cutaneous lewisite exposure. These significantly altered genes are further subjected to clustering analysis or categorization into Th1-, Th2-, or Th17-related responses. Using the SABiosciences online RT Profiler PCR Array data analysis software, the cluster diagram of inflammatory genes was generated. The genes with higher correlation coefficients across different samples are clustered together by rows. Thus, samples within the same cluster represent closer gene expression patterns than samples in different clusters. In this regard, the clustering patterns of the samples fell into two groups, with nonexposed skin (NS) clustered in one group and distal skin (DS), adjacent skin (AS), and lewisite-exposed skin (LES) clustered in another group. Each row represents a single gene labeled with the gene name, whereas each column represents an independent skin samples. The color in each cell reflects the relative gene expression level of the corresponding sample. The color scale at the bottom of the diagram indicates the magnitude of gene expression. Expression levels greater than the mean are shaded in red and those below the mean are shaded in green. B: Western blot showing the expression of IL-1 13 in NS, DS, AS, and LES. C: qPCR showing the expression of Il1b, Il6, Tnf, and Tgfb1 in NS, DS, AS, and LES. Data are expressed as means \pm SEM of three independent samples. $* * * P<0.001$ versus NS. avg, average; max, maximum; min, minimum.
} 
lewisite-exposed skin and adjacent skin were further subclustered together (Figure 3A). These data suggest that although inflammatory responses in the lesional skin were much more severe, distal skin areas also have some effect.

The constellation of cytokines are believed to act in concert to produce tissue injury and regulate recovery process. ${ }^{17}$ Loss of equilibrium between proinflammatory and anti-inflammatory cytokines or chemokines and/or their receptor-mediated responses determine the magnitude of tissue damages and tissue repair. ${ }^{18}$ We therefore broadly assessed the expression of genes, which could be categorized into Th1-, Th2-, and Th17-related responses (Figure 3A and Supplemental Table S2). Lewisite treatment resulted in a marked induction of Th1-related markers and a reduction of Th2-related markers. Of 15 Th1 genes, the mRNA levels of nine genes, including Il1b, Spp1, Ccl4, Ccr1, Ccr5, Ccl5, Cxc15, Cxcl10 (I18), and Cxcl11, were significantly up-regulated in lewisite-exposed skin (Figure 3A and Supplemental Table S2). Illb is a wellknown proinflammatory cytokine and a classic marker of cutaneous inflammation. ${ }^{19}$ Its expression was induced by 3.4-fold in lewisite-exposed skin when compared with nonexposed skin (Supplemental Table S2). The induction of Illb was further confirmed by Western blot and qPCR analysis (Figure 3, B and C). However, the expression of Ccl5 and Cxcl11 was increased by 17.0- and 11.9-fold, respectively, by lewisite (Supplemental Table S2). Interestingly, interaction of $\mathrm{Ccl} 5$ with its receptor Ccr5 was found to play a pivotal role in mouse wound-healing process. ${ }^{20}$ Cxcl11 shares its receptor Cxcr3 with Cxcl10 in recruitment of activated $\mathrm{T}$ cells. Recent studies indicate that CXCR3 signaling plays a critical role in the process of cutaneous wound healing. ${ }^{21}$ The classic Th1 marker Ifng was not altered significantly. Surprisingly, the expression of Tnf was decreased by fivefold in lewisite-exposed skin (Supplemental Table S2), which was also confirmed by qPCR (Figure 3C). This finding is consistent with earlier report that Tnf was decreased by lewisite treatment in human keratinocytes. ${ }^{22}$ Unlike Th1-related markers, most Th2-related markers, including Il15, Il13ra, Il10rb, Il10ra, Ccl1, Ccl17, Ccl22, Ccr4, and Ccr8, were down-regulated. Notably, Ccl22, Ccr4, Ccl1, and Ccl17 were downregulated by 27-, 23-, 14-, and 10-fold, respectively, in lewisite-exposed skin compared with nonexposed skin (Figure 3A and Supplemental Table S2). In this regard, the protein encoded by $\mathrm{Ccl} 22$ is primarily secreted by macrophages and is known to elicit its effects on target cells by interacting with Ccr4, a chemokine receptor that is also required for $\mathrm{Ccl} 17$ function. ${ }^{23}$ However, the expression of the other Th2-type cytokines Il10 and Il4 were only modestly altered in lewisite-exposed skin (Supplemental Table S2).

The expression of Th17-related markers was affected by lewisite challenge. On one hand, compared with nonexposed skin, 2- to 11-fold increased expression was identified for Il6, Il20, Il17b, Cc17, Cxcl1, Cxcl13, and Cxcl5 in lewisite-exposed skin. Il6 is associated with acute-phase response and is considered to be proinflammatory under multiple pathophysiologic conditions. ${ }^{24}$ Compared with nonexposed skin, Il6 was induced by 11-fold in lewisiteexposed skin, which was also confirmed by qPCR (Figure 3C and Supplemental Table S2). We found a sixfold increase in the expression of Il20 relative to nonexposed skin (Supplemental Table S2), which is a Th17 cytokine considered important in the regulation of cutaneous inflammation, keratinocytes proliferation, and differentiation. ${ }^{25}$ However, the cytokines or chemokines Ill $\alpha$, Il15, Ccr6, Il1f8, Ccl20, and Il1f6, which are involved in inflammatory diseases, such as psoriasis, ${ }^{26}$ were found to be significantly down-regulated. Il1f6 was reduced by 100 -fold in lewisite-exposed skin (Supplemental Table S2). We also observed down-regulation of the anti-inflammatory gene Tgfb1 by qPCR in lewisite-exposed skin. Interestingly, some of these changes in cytokines, chemokines, or receptor gene were not only observed in lewisite-exposed skin but also in adjacent skin and distal skin, suggesting the possibility that these inflammatory responses are mediated by the systemic effects of lewisite (Figure 3A and Supplemental Table S2). Detailed investigation of pathways regulating these inflammatory genes will reveal exact molecular signatures of lewisite-induced inflammation.

\section{Potential Signaling Pathways Underlying Lewisite- Mediated Cutaneous Toxicity}

To investigate molecular mechanism underlying the pathogenesis of lewisite-induced skin lesions, we used a multiplepathway activity assay (Cignal Finder 45-Pathway Reporter Array). We again used PAO to investigate the signaling pathways induced in human skin keratinocytes. Of 45 reporters in this array, PAO significantly induced luciferase activity $(P<0.05)$ of 28 reporters by $>1.5$-fold 6 hours after treatment compared with vehicle-treated control (Supplemental Figure S2A). Interestingly, ERSE, AARE, and ATF6 reporters, which are related to ER stress, were significantly enhanced by more than twofold (Supplemental Figure S2A). Similarly, transcriptional factors involved in oxidative stress (ARE and AP1), inflammatory/immune responses (VDR, AP1, GAS, and STAT3), and DNA damage/apoptosis (AP1 and p53) were also activated by PAO treatment (Supplemental Figure S2A). We are further evaluating the description of some of these pathways, which is beyond the scope of this article.

\section{Lewisite Treatment Leads to UPR Induction and Oxidative Stress in Murine Skin Keratinocytes}

Because we observed that PAO enhanced ER stress-related UPR regulatory transcriptional factor reporter activities, we next tested whether lewisite induces UPR signaling in $\mathrm{HaCaT}$ cells for which cell viability and half maximal 
A

Effect of Lewisite on HaCaT Viability (MTS, $6 \mathrm{~h}$ )

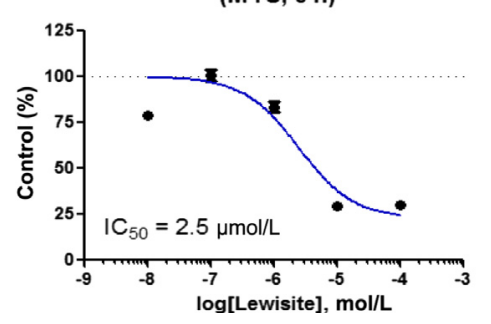

C

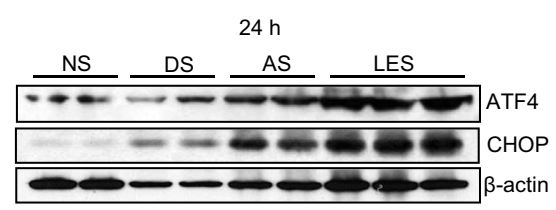

$\mathbf{E}$

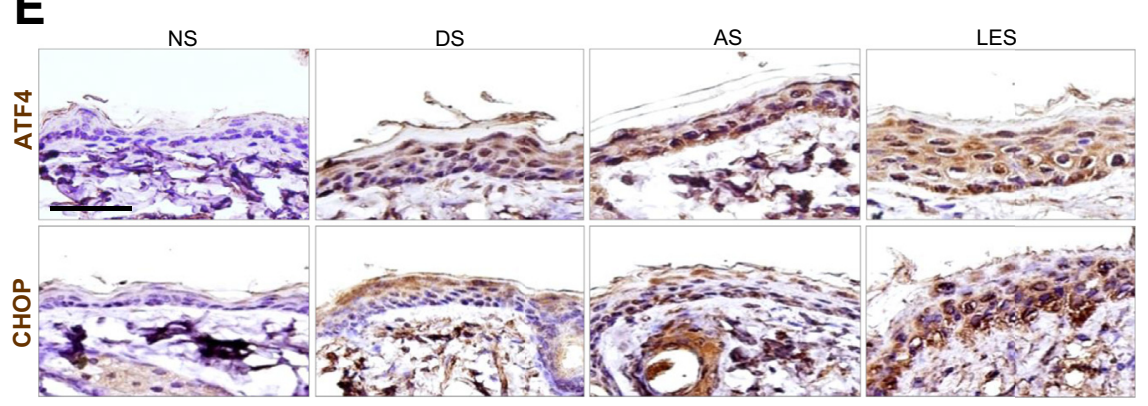

$\mathbf{F}$
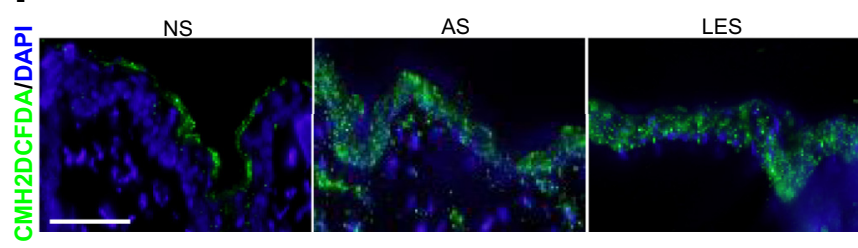
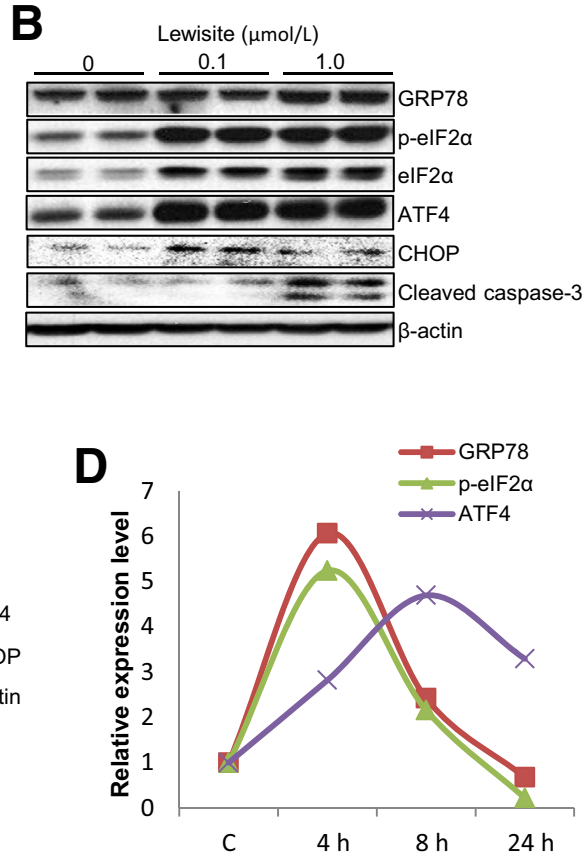

Figure 4 Lewisite induces unfolded protein response (UPR) signaling both in vivo and in vitro. A: Graph showing HaCaT cell viability after treatment with lewisite for 6 hours as assessed by MTS assay. B: Western blot analysis showing the induction of UPR regulatory proteins GRP78, phosphoeIF2 $\alpha$ (p-eIF2 $\alpha$ ), eIF2 $\alpha$, ATF4, CHOP, and cleaved caspase- 3 in $\mathrm{HaCaT}$ cells treated with lewisite for 6 hours. C: Western blot analysis showing expression of ATF4 and CHOP in nonexposed skin (NS), distal skin (DS), adjacent skin (AS), and lewisite-exposed skin (LES). D: Densitometry analysis showing the time-dependent induction of UPR proteins, including GRP78, p-eIF2 $\alpha$, and ATF4. For the control, 4-hour, and 8-hour groups, two independent skin samples were analyzed; for the 24-hour group, three independent skin samples of LES are analyzed. E: Images showing the immunohistochemical staining of CHOP and ATF4 in NS, DS, AS, and LES. F: Images showing fluorescence of oxidized CMH2DCFDA in NS, AS, and LES. Data are presented as mean only (D). Scale bars $=25 \mu \mathrm{m}$ (E and $\mathbf{F})$. ATF4, activating transcription factor $4 ; \mathrm{C}$, control; CHOP, CCAAT/enhancer-binding protein (C/EBP) homologous protein; $\mathrm{IC}_{50}$, half maximal inhibitory concentration. inhibitory concentration of lewisite were determined. The half maximal inhibitory concentration of lewisite was 2.5 $\mu \mathrm{mol} / \mathrm{L}$ for a 6-hour treatment (Figure 4A). Using two concentrations of lewisite $(0.1$ and $1.0 \mu \mathrm{mol} / \mathrm{L}$ at 6 hours $)$, we observed a significant dose-dependent up-regulation of the UPR marker proteins GRP78, p-eIF2 $\alpha$, ATF4, and CHOP (Figure 4B) but changes in ATF6 and spliced XBP1 mRNA were not significant (data not shown). Up-regulation of UPR signaling was also accompanied with induction of apoptosis, particularly at higher concentrations (Figure 4B).

Next, we investigated whether lewisite induces UPR signaling in murine skin. We treated $P t c h 1^{+/-} / S K H-1$ mice topically with $1.5 \mathrm{mg} / \mathrm{kg}$ of lewisite (approximately one-tenth of $\left.\mathrm{LD}_{50}\right){ }^{27}$ ATF4 and CHOP expression was highly induced in the skin of lewisite-treated mice at 24 hours after treatment (Figure 4C). Despite the increase in
ATF4 and CHOP, the phosphorylation of eIF2 $\alpha$ was found to be reduced at this time point. Similar reduction was also observed in the expression of GRP78 (Supplemental Figure S2B). Because phosphorylation of eIF2 $\alpha$ is an upstream event in the induction of ATF4 and CHOP, we then determined the kinetics of UPR signaling induction incorporating earlier time points after lewisite treatment. In these samples, the expression of GRP78 and phospho-eIF2 $\alpha$ was elevated at 4 hours but returned to control level by 8 hours, whereas ATF4 remained constantly up-regulated between 4 and 24 hours (Figure 4D and Supplemental Figure S2C) as also confirmed by IHC staining (Figure 4E). Using the fluorescent probe CM-H2DCFDA, we found a marked increase of ROS production in both lewisite-exposed skin and adjacent skin at 24 hours after treatment (Figure 4F). 

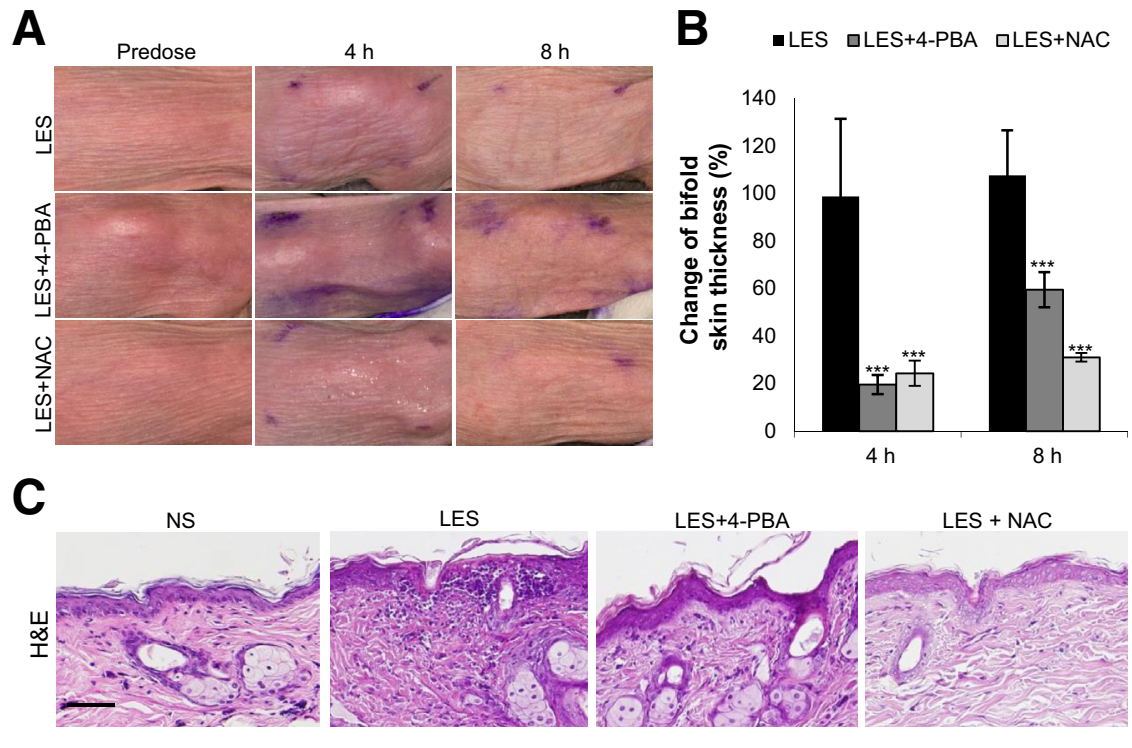

D
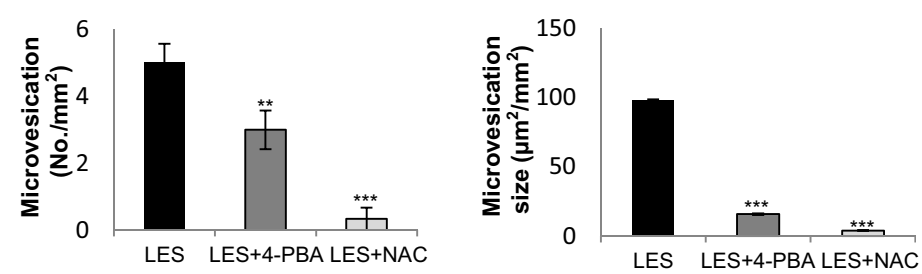

E

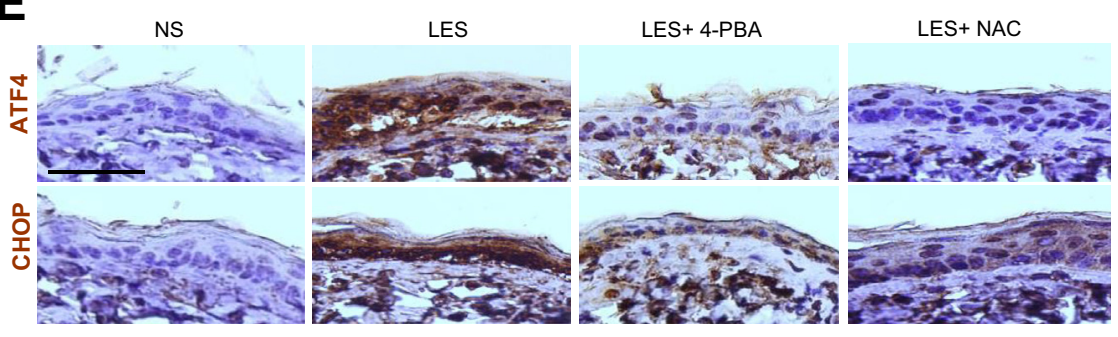

$\mathbf{F}$

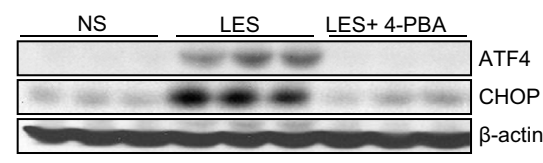

G

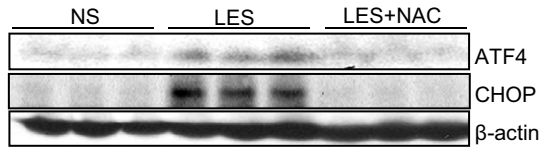

Figure 5 Effect of 4-phenylbutyric acid (4PBA) and $N$-acetylcysteine (NAC) on lewisiteinduced skin damage and unfolded protein response (UPR) signaling in $P$ tch $1^{+/-} /$SKH-1 mice. Ptch $1^{+/-} / \mathrm{SKH}-1$ mice were treated topically with vehicle or $2.0 \mathrm{mg} / \mathrm{kg}$ of lewisite in combination with $4 \mathrm{mg}$ of 4-PBA per mouse or $5 \mathrm{mg}$ of NAC per mouse in an area of $1.6 \times 1.6 \mathrm{~cm}^{2}$ and observed up to 8 hours. To reduce the number of animals, the vehicle group and lewisite group serve as negative and positive control for both the 4-PBA and NAC studies. A: Images showing gross skin changes of dose site skin. Representative images of female mice are shown. B: Bar diagram showing skin bifold thickness. C: Representative images showing the histologic findings of the dose-site skin. D: Bar diagram showing the count of microvesications. The number and size of microvesications were plotted against unit area. E: Immunohistochemical staining showing expression of ATF4 and CHOP in dose-site skin. $\mathbf{F}$ and $\mathbf{G}$ : Western blot analysis of UPR regulatory proteins ATF4 and CHOP in dose site skin. Data are expressed as means \pm SEM of six animals. $* * P<0.01,{ }^{* * *} P<0.001$ versus the LES group. Scale bars: $25 \mu \mathrm{m}(\mathbf{E}) ; 50 \mu \mathrm{m}$ (C). ATF4, activating transcription factor 4; CHOP, CCAAT/enhancerbinding protein (C/EBP) homologous protein; $H \& E$, hematoxylin and eosin; LES, lewisiteexposed skin; NS, nonexposed skin.
Chemical Chaperone 4-PBA Attenuates LewisiteInduced Skin Damage and Inflammation by Blocking UPR Induction

To demonstrate that UPR signaling underlies lewisiteinduced skin damage, we used small molecules-based approaches. We initially tested the chemical chaperone 4-PBA, which is known to ease ER stress under various experimental settings. ${ }^{28}$ Treatment with 4-PBA reduced PAO-induced ER stress-related transcription factor reporter activities in human skin keratinocytes (Supplemental Figure S3A). In addition, lewisite-mediated reduction in cell viability was restored by 4-PBA (Supplemental Figure S3B). For confirming these data in vivo, 4-PBA was administered topically to $\mathrm{Ptch}^{+/-} / \mathrm{SKH}-1$ mice 5 minutes after lewisite treatment, which significantly decreased skin damage as characterized by reduction in skin bifold thickness, inflammatory cell infiltration, and microvesication (Figure 5, A-D). Skin sections revealed reduced pyknosis, karyolysis, cytoplasmic eosinophilia, and vacuolation in epidermal keratinocytes (Figure 5C). Furthermore, these changes significantly correlated with the potential of 4-PBA to block lewisiteinduced UPR signaling. A significant reduction in UPR regulatory proteins ATF4 and CHOP could be seen (Figure 5, E and F, and Supplemental Figure S3C), which was accompanied by diminished ROS generation (Figure 6A) and decreased expression of the proinflammatory cytokines $\mathrm{Illb}$ and $\mathrm{Il} 6$ and the $\mathrm{PGE}_{2}$ level (Figure 6, B and C).

UPR signaling regulates inflammation through NF- $\kappa \mathrm{B}$ and JNK signaling in various disease models. ${ }^{10} \mathrm{~A}$ 


\section{A}

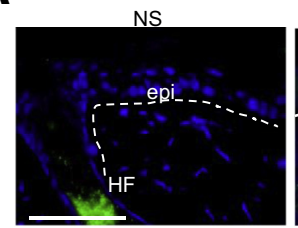

B

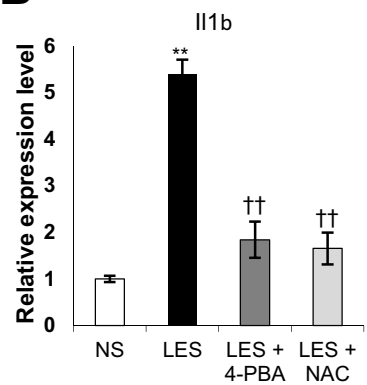

D

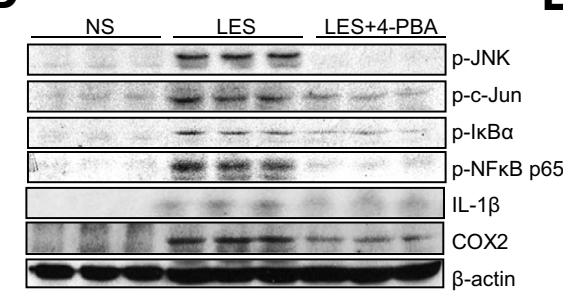

$\mathbf{F}$
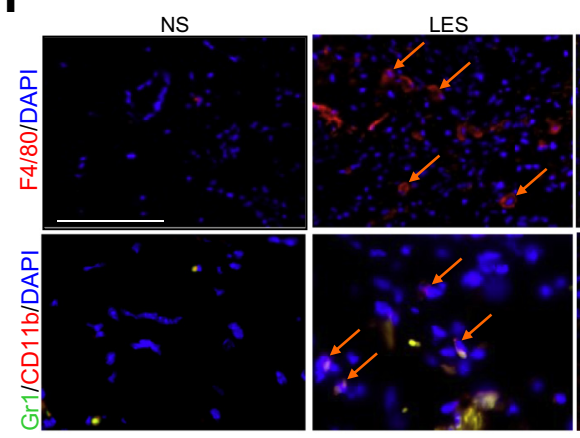

G

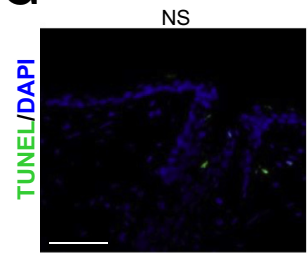

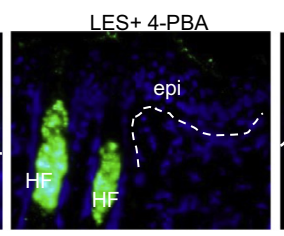

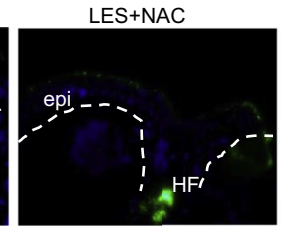

C

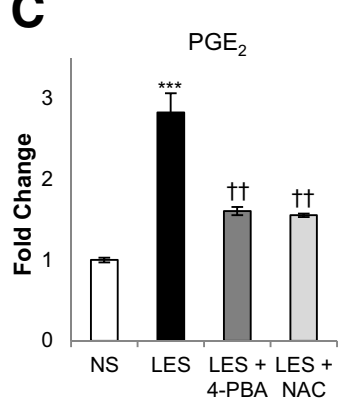

E

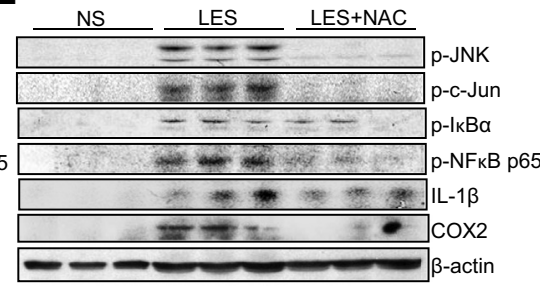

LES+4-PBA
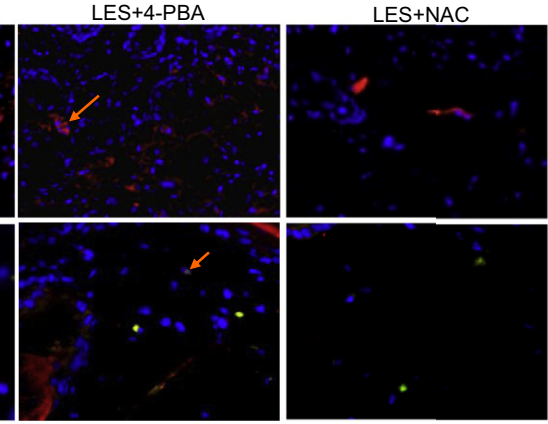

LES+4-PBA

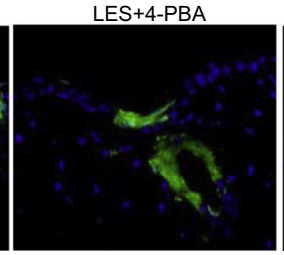

LES+NAC

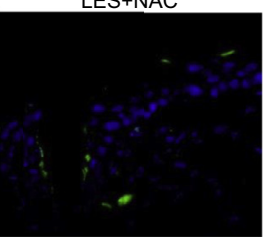

Figure 6 Effect of 4-phenylbutyric acid (4-PBA) and $\mathrm{N}$-acetylcysteine (NAC) on lewisite-induced cutaneous inflammation and cell death in $P$ tch $1^{+/-}$ SKH-1 mice. A: Images showing fluorescence of oxidized CM-H2DCFDA in the epidermis (epi) (dotted lines) compared with the dermis (area below the dotted lines). B: qPCR showing the expression of Il1b and Il6 mRNA in the skin. C: Bar diagram showing the fold-change of prostaglandin $E_{2}\left(P E_{2}\right)$ level in skin lysates. D and E: Western blot and densitometry analysis of the expression of phosphoJNK ( $p-J N K)$, phospho-c-Jun ( $p-c-J u n)$, phospho-

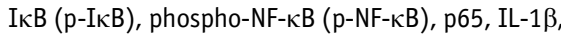
and cyclooxygenase-2 (COX2) in skin. F: Immunofluorescence staining of inflammatory cell surface markers Gr1/CD11b and F4/80 in the skin. Orange arrows indicate the staining for Gr1/CD11b or F4/ 80. G: Representative images showing terminal deoxynucleotidyl transferase-mediated dUTP nickend labeling (TUNEL) staining in the skin. Data are expressed as means \pm SEM of three independent samples. ${ }^{*} P<0.01,{ }^{* * *} P<0.001$ versus nonexposed skin (NS); ${ }^{\dagger \dagger} P<0.01$ versus lewisite-exposed skin (LES). Scale bars: $25 \mu \mathrm{m}$ (A and F); $50 \mu \mathrm{m}$ (G). $\mathrm{H} \& \mathrm{E}$, hematoxylin and eosin; HF, hair follicle. profound decrease in lewisite-mediated $\mathrm{NF}-\kappa \mathrm{B}$ and $\mathrm{JNK}$ signaling-related phospho-JNK, phospho-c-Jun, phospho-I $\kappa$ B, phospho-NF- $\kappa$ B p65, IL-1 $\beta$, and $\mathrm{COX} 2$ could be observed in the skin of mice receiving 4-PBA (Figure 6D and Supplemental Figure S3D). These effects were associated with the marked decreases in the infiltration of proinflammatory neutrophils $\left(\mathrm{Gr}^{+}\right.$and $\mathrm{CD}_{11 \mathrm{~b}^{+}}$) and macrophages $\left(\mathrm{F} 4 / 80^{+}\right.$) (Figure 6F). In addition, 4-PBA afforded protection against lewisiteinduced apoptosis in the skin. However, these protective effects were more pronounced in the area of interfollicular epidermis because apoptotic cells could still be observed in areas around hair follicle-associated keratinocytes (Figure 6G).

\section{Lewisite-Mediated ROS Generation Induces Cutaneous UPR Signaling}

We observed a persistent up-regulation of ROS and UPR signaling at the lewisite dosing-site skin between 8 and 24 hours (Figure 4F and Figure 6A). Consistently, in the luciferase reporter assay, we observed that antioxidant NAC 
significantly reduced PAO-induced ER stress in human keratinocytes (Supplemental Figure S3A). Furthermore, treatment with NAC also restored lewisite-mediated impairment in keratinocyte viability, supporting the role of ROS in cell death (Supplemental Figure S3B). Because ROS is known to the activate UPR signaling pathway, ${ }^{10}$ we decided to unravel whether lewisite-induced UPR signaling in the skin is mediated via ROS generation. Topical treatment with antioxidant NAC (5 minutes after lewisite treatment) not only blocked ROS production (Figure 6A) but also resulted in significant diminution of UPR signaling as demonstrated by decreased expression of ATF4 and CHOP (Figure 5, E and G, and Supplemental Figure S3E). Significantly, NAC-mediated decrease in UPR signaling was also associated with its ability to protect against lewisite-induced skin lesions, including inflammation and bifold skin thickness (Figure 5, A-C). Notably, $90 \%$ of microvesication formed in the lewisite treatment group disappeared after NAC treatment (Figure 5D). Microscopic examination of tissue sections revealed significantly reduced pyknosis, karyolysis, cytoplasmic eosinophilia, and vacuolation of lewisite-treated epidermal keratinocytes by NAC (Figure 5C). NAC also afforded similar protection against lewisite-induced inflammatory cell infiltration (Figure 5C and Figure 6F), proinflammatory cytokines (Illb and Il6), and $\mathrm{PGE}_{2}$ level (Figure 6, B and C). Mechanistically, NAC abrogated lewisite-induced NF- $\kappa \mathrm{B}$ and JNK pathways as indicated by the decreased expression of phospho-JNK, phospho-c-Jun,

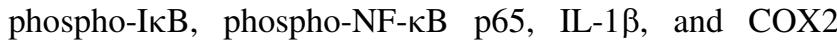
(Figure 6E and Supplemental Figure S3F), suggesting a nice cascading effect of ROS, UPR inflammation, and cell death by lewisite. NAC seems to be more effective in downregulating Il6 compared with 4-PBA (Figure 6B), and unlike 4-PBA, NAC reduced apoptosis in both interfollicular and follicular epidermal keratinocytes (Figure 6G).

\section{Discussion}

Lewisite is an arsenic-based chemical warfare agent that can still be a threat from storage, destruction, or deliberate use by terrorists against civilian populations. Therefore, defining its mechanism of action and molecular target-based therapy is necessary. In addition, to understand the pathogenesis of these chemicals in humans, a suitable animal model that could faithfully recapitulate human pathogenesis is also required. In this study, we identified the $P t c h 1^{+/-} / S K H-1$ hairless mouse as a suitable murine model, which is highly sensitive and recapitulates many features of lewisite-induced skin lesions in humans. ${ }^{4,7}$ Additional relevance of the Ptch $1^{+/} /$SKH-1 hairless model is based on the fact that sonic hedgehog signaling is often induced after tissue injury for wound healing and/or for tissue repair or remodeling. ${ }^{29}$

Our observations that inflammatory responses in lewisiteinjured skin are distinct from those reported for mustard agents suggest that two chemicals act through distinct mechanisms. In lewisite-challenged skin, significant induction of Illb and Il6 with down-regulation of Tnf and Ill $\alpha$ and no significant alterations in Ifng, Il4, and Il10 characterized the injury. However, mustards are known to induce Ill $\alpha$, Illb, Il6, Tnf, and Ifng, while suppressing Il4 and Il10. ${ }^{22,30}$ Although pointing to a distinct mechanism underlying inflammatory responses by the two classes of vesicants, these data also highlight their potential value as biomarkers in distinguishing vesicant exposure in the battle field. In addition, lewisite-induced alterations in many of the inflammatory cytokines or chemokines not only were limited to the site of skin exposure but also could be observed in adjacent skin and distal skin. These rather scattered effects of lewisite may be related to the earlier described lewisite shock or may be attributable to the diffusion of lewisite across the larger tissue area after its cutaneous absorption.

Our data indicating that lewisite activates UPR signaling by generating ROS provide a novel mechanism by which lewisite induces inflammation and blistering in the exposed skin. However, it remains unclear how lewisite initiates ER stress. It is known that the arsenic (III) species by binding to the cysteine residues of unfolded proteins can impair their folding ability, ultimately leading to the accumulation of unfolded and/or misfolded proteins ${ }^{31}$ and to the activation of UPR signaling. ${ }^{32}$ Earlier, we also found that the UPR pathway underlies the pathogenesis of subacute arseniteinduced mild inflammatory responses in the skin. ${ }^{13}$ Therefore, together this provides a likely scenario that trivalent arsenic released from metabolic degradation of lewisite may play a significant role in the induction of ER stress. Interestingly, ER stress-regulated pathogenesis is not unique to the toxic manifestations of arsenicals but is important in the pathobiology of multiple diseases. ${ }^{11}$ Our observations that the chemical chaperon 4-PBA provides protection against lewisite-induced UPR signaling, inflammation, blistering, and cell death in the skin of $P t c h 1^{+/-} / S K H-1$ mice further confirm the pivotal role of UPR signaling in the development of the skin lesions after lewisite exposure. However, based on the in vitro data from the reporter array, it is likely that other pathways may also be involved in lewisiteinduced skin injury.

Our observations that NAC blocks lewisite-mediated ROS production with a concomitant attenuation of skin injury and associated UPR pathway suggest an upstream role of ROS in these events. The mechanism by which NAC affords protection against lewisite-induced injury is not clear. However, in addition to its antioxidant potential, it may act via augmenting the biosynthesis of glutathione and thus may expand the intracellular pool of thiols for arsenic (III) binding or chelating, ${ }^{33}$ thereby facilitating its metabolic dissemination and fast excretion and ultimately leading to reduced toxicity.

Considering rapid reactivity of lewisite, determination of a therapeutic window of 4-PBA and NAC would be a highly significant and necessary aspect of future translational 
studies. In this study, we tested only a short 5-minute window. Our work thus serves as a proof-of-principle study to reveal that targeting UPR signaling or blocking ROS production after lewisite exposure may contribute significantly to reducing cutaneous toxicity of such reactive vesicants. Studies in our laboratory are now focused on defining a broad therapeutic window up to 2 hours for these new series of antidotes identified here. The advantages of these chemicals are that they are approved by the US Food and Drug Administration for urea cycle disorders and acetaminophen overdose toxicity, respectively. Therefore, repurposing them as antidotes for the toxic effects of arsenicals could be relatively easier.

\section{Supplemental Data}

Supplemental material for this article can be found at http://dx.doi.org/10.1016/j.ajpath.2016.06.012.

\section{References}

1. Gupta RC: Handbook of Toxicology of Chemical Warfare Agents. Philadelphia, PA, Elsevier, 2009

2. Marrs TC, Maynard RL, Sidell FR: Chemical Warfare Agents: Toxicology and Treatment. Hoboken, NJ, John Willey \& Sons, 2007

3. Watson AP, Griffin GD: Toxicity of vesicant agents scheduled for destruction by the Chemical Stockpile Disposal Program. Environ Health Perspect 1992, 98:259-280

4. McGown EL, Ravenswaay Tv, Damlao CR, O'Connor RJ, Black KE: Histologic changes caused by application of lewisite analogs to mouse skin and human skin xenografts. Proceedings of the Fifth Annual Chemical Defense Bioscience Review 1985, 941.

5. Greaves IA, Koelle GB, Koller LD, Krieger RI, Saady JJ, Stevens DK, Walker BJ, Watson AP: Guidelines for Chemical Warfare Agents in Military Field Drinking Water. Washington, DC, National Academies Press, 1995

6. Sidell FR, Takafuji ET, Franz DR: Textbook of Military Medicine: Medical Aspects of Chemical and Biological Warfare. Phoenix, AZ, TMM Publications, 1997

7. Davis MIJ: The dermatologic aspects of the vesicant war gases. JAMA 1944, 126:5

8. Vilensky JA, Redman K: British anti-Lewisite (dimercaprol): an amazing history. Ann Emerg Med 2003, 41:378-383

9. Mouret S, Wartelle J, Emorine S, Bertoni M, Nguon N, CleryBarraud C, Dorandeu F, Boudry I: Topical efficacy of dimercaptochelating agents against lewisite-induced skin lesions in SKH-1 hairless mice. Toxicol Appl Pharmacol 2013, 272:291-298

10. Zhang K, Kaufman RJ: From endoplasmic-reticulum stress to the inflammatory response. Nature 2008, 454:455-462

11. Cao SS, Kaufman RJ: Endoplasmic reticulum stress and oxidative stress in cell fate decision and human disease. Antioxid Redox Signal 2014, 21:396-413

12. Chaudhary SC, Tang X, Arumugam A, Li C, Srivastava RK, Weng Z, Xu J, Zhang X, Kim AL, McKay K, Elmets CA, Kopelovich L, Bickers DR, Athar M: Shh and p50/Bc13 signaling crosstalk drives pathogenesis of BCCs in Gorlin syndrome. Oncotarget 2015, 6: 36789-36814

13. Li C, Xu J, Li F, Chaudhary SC, Weng Z, Wen J, Elmets CA, Ahsan H, Athar M: Unfolded protein response signaling and MAP kinase pathways underlie pathogenesis of arsenic-induced cutaneous inflammation. Cancer Prev Res (Phila) 2011, 4:2101-2109
14. Nguon N, Clery-Barraud C, Vallet V, Elbakdouri N, Wartelle J, Mouret S, Bertoni M, Dorandeu F, Boudry I: Time course of lewisite-induced skin lesions and inflammatory response in the SKH-1 hairless mouse model. Wound Repair Regen 2014, 22:272-280

15. Benavides F, Oberyszyn TM, VanBuskirk AM, Reeve VE, Kusewitt DF: The hairless mouse in skin research. J Dermatol Sci 2009, 53:10-18

16. Rice P, Brown RF: The development of Lewisite vapour induced lesions in the domestic, white pig. Int J Exp Pathol 1999, 80:59-67

17. Steinman L: A brief history of $\mathrm{T}(\mathrm{H}) 17$, the first major revision in the $\mathrm{T}(\mathrm{H}) 1 / \mathrm{T}(\mathrm{H}) 2$ hypothesis of $\mathrm{T}$ cell-mediated tissue damage. Nat Med 2007, 13:139-145

18. Butterfield TA, Best TM, Merrick MA: The dual roles of neutrophils and macrophages in inflammation: a critical balance between tissue damage and repair. J Athl Train 2006, 41:457-465

19. Keyel PA: How is inflammation initiated? Individual influences of IL-1, IL-18 and HMGB1. Cytokine 2014, 69:136-145

20. Ishida Y, Kimura A, Kuninaka Y, Inui M, Matsushima K, Mukaida N, Kondo T: Pivotal role of the CCL5/CCR5 interaction for recruitment of endothelial progenitor cells in mouse wound healing. J Clin Invest 2012, 122:711-721

21. Huen AC, Wells A: The beginning of the end: CXCR3 signaling in latestage wound healing. Adv Wound Care (New Rochelle) 2012, 1:244-248

22. Arroyo CM, Burman DL, Kahler DW, Nelson MR, Corun CM, Guzman JJ, Smith MA, Purcell ED, Hackley BE Jr, Soni SD, Broomfield CA: TNFalpha expression patterns as potential molecular biomarker for human skin cells exposed to vesicant chemical warfare agents: sulfur mustard (HD) and Lewisite (L). Cell Biol Toxicol 2004, 20:345-359

23. Katou F, Ohtani H, Nakayama T, Ono K, Matsushima K, Saaristo A, Nagura H, Yoshie O, Motegi K: Macrophage-derived chemokine (MDC/CCL22) and CCR4 are involved in the formation of $\mathrm{T}$ lymphocyte-dendritic cell clusters in human inflamed skin and secondary lymphoid tissue. Am J Pathol 2001, 158:1263-1270

24. Heinrich PC, Castell JV, Andus T: Interleukin-6 and the acute phase response. Biochem J 1990, 265:621-636

25. Rich BE, Kupper TS: Cytokines: IL-20-a new effector in skin inflammation. Curr Biol 2001, 11:R531-R534

26. Nedoszytko B, Sokolowska-Wojdylo M, Ruckemann-Dziurdzinska K, Roszkiewicz J, Nowicki RJ: Chemokines and cytokines network in the pathogenesis of the inflammatory skin diseases: atopic dermatitis, psoriasis and skin mastocytosis. Postepy Dermatol Alergol 2014, 31:84-91

27. Williams MA, Reddy G, Quin MJ, Johnson MS: Wildlife Toxicity Assessments for Chemicals of Military Concern. Philadelphia, PA, Elsevier, 2015

28. Srivastava RK, Li C, Chaudhary SC, Ballestas ME, Elmets CA, Robbins DJ, Matalon S, Deshane JS, Afaq F, Bickers DR, Athar M: Unfolded protein response (UPR) signaling regulates arsenic trioxidemediated macrophage innate immune function disruption. Toxicol Appl Pharmacol 2013, 272:879-887

29. Asai J, Takenaka H, Kusano KF, Ii M, Luedemann C, Curry C, Eaton E, Iwakura A, Tsutsumi Y, Hamada H, Kishimoto S, Thorne T, Kishore R, Losordo DW: Topical sonic hedgehog gene therapy accelerates wound healing in diabetes by enhancing endothelial progenitor cell-mediated microvascular remodeling. Circulation 2006, 113:2413-2424

30. Sawale SD, Ambhore PD, Pawar PP, Pathak U, Deb U, Satpute RM: Ameliorating effect of S-2(omega-aminoalkylamino) alkylaryl sulfide (DRDE-07) on sulfur mustard analogue, 2-chloroethyl ethyl sulfideinduced oxidative stress and inflammation. Toxicol Mech Methods 2013, 23:702-710

31. Ramadan D, Rancy PC, Nagarkar RP, Schneider JP, Thorpe C: Arsenic(III) species inhibit oxidative protein folding in vitro. Biochemistry 2009, 48:424-432

32. Hunt KM, Srivastava RK, Elmets CA, Athar M: The mechanistic basis of arsenicosis: pathogenesis of skin cancer. Cancer Lett 2014, 354:211-219

33. Delnomdedieu M, Basti MM, Otvos JD, Thomas DJ: Transfer of arsenite from glutathione to dithiols: a model of interaction. Chem Res Toxicol 1993, 6:598-602 